\title{
Effects on the Calibration of Instruments in Surface Metrology
}

\author{
Özgür Tan \\ oezguer.tan@ingenieur.de
}

\begin{abstract}
:
Thanks to the improvements in manufacturing techniques, it is becoming more common to alter the functional performance of products by controlling the geometrical properties of technical surfaces. The required characterization of surfaces can be done by using different optical measurement techniques. In order to have reliable measurement results, one of the pre-requisite is the proper calibration of the instrument. Although new ideas, like 3D Siemens-Star are becoming available in surface metrology, complexity of optical measurement techniques makes it not easy to calibrate them. In this study, depending on the available guidelines, some effects on the calibration of optical measurement techniques are given.
\end{abstract}

Key words: Surface metrology, calibration, Siemens-star, optical methods

\begin{abstract}
1 Introduction
Development of surface metrology is traditionally based on the tactile methods, which acquire the surface data by contacting on the surface itself. Although for most of the applications, this type of analyzing is enough, tactile methods are generally time consuming and they may damage the workpiece during data acquisition. Furthermore due to the limitations of the shape and the size of the stylus, structural properties of the surfaces can not always be illustrated. They are not capable to penetrate into the structure of the surfaces.
\end{abstract}

Thanks to the latest developments in measurement techniques, there are now many optical methods to characterize technical surfaces. In comparison to surface profile measurements (like tactile ones), optical methods mostly provide areal information which make them statically more reliable and more representative for the investigated topography. Furthermore in most cases they are faster than the available tactile techniques.

However there are some issues which should be taken into account in order to apply such techniques properly. The most important metrological point to get traceable results, is the calibration of instruments. Although optical techniques provide great possibilities, due to the complexity of their working principle, calibration process is not such straightforward.
Depending on the applied technique, different issues should be taken into account. And it is crucial to follow the available standards and guidelines during calibration process.

\section{Guidelines and Standards}

Although there is a great effort to cover the three dimensional measurement of surface texture by international standards, it is not completely achieved at this stage. Main development activities are done by the research projects in Europe and other countries and the relevant standards, like ISO 25178 are being considered by ISO TC 213.

Mainly in all guidelines like VDI 2655 [1] the following metrological properties are investigated: measurement noise, flatness, orthogonality, movement of the axes and resolution. In such investigations different types of standards are required, see figure 1.

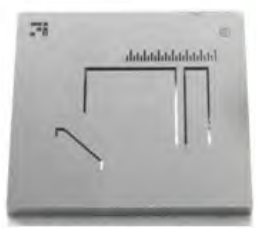

Depth Standard

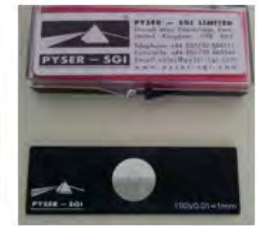

Stage micrometer

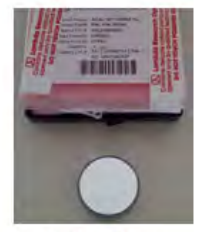

Optical flat
Fig. 1. Overview of some standards used in surface metrology

Calibration of the horizontal axis is performed by a stage micrometer, whereas optical flat is 
used to estimate the instrument noise. As stated in [1] after having measured the optical flat in different positions, the acquired topographies are averaged, storaged and subtracted in further measurements. By this way reference plane can be calculated e.g. for the confocal techniques. Different types of vertical standards (step height standard) can be used to characterize the vertical properties. Although such metrological properties are essential to characterize the instruments and to have traceable results, they cannot completely describe the behavior of the system for the measurement of technical surfaces. Technical surfaces are different from idealized ones (like the ones shown in figure 1) and the mentioned methods are not always enough to have a reliable system. The conditions under which the calibration is performed may not be relevant to the conditions of the real measurement task.

In order to investigate the performance of the instrument for the "real task", there exist some capability analysis tests, like MSA [2]. Due to the simplicities in the methods of capability analysis, many companies, especially automotive sector, have set it as standard and use it uniformly in most of the production locations. Besides simplicity, with these methods not only the capability of the measurement device can be analyzed but they can also be applied for pre-acceptance test at the manufacturer before delivery. Since they are performed on-site, they also represent the environmental conditions very well. But capability analyses include only short term influences, because they are performed in a well defined time period. So it is necessary to control the stability, which requires additional monitoring at regular intervals. Another important point is the fact that, such capability analyses are performed without considering the working principle of the applied technique. Especially in the complex techniques, like the optical ones, this approach should be applied more carefully.

As a sum up, characterization of optical techniques requires the application of guidelines and tests but it is important to understand the limitations of the techniques. In this study some of such conditional effects are shown and tried to make the end user be aware of such effects.

\section{Some of the Effects on the Calibration of the Instruments}

Mentioned methods and guidelines like [1] show the ways to characterize the metrological properties of the instruments however they do not completely deal with the real measurement task. In order to highlight the additional requirements during the calibration and application of such methods, the following issues are considered throughout this study. They do not cover all effects but given as examples.

\subsection{Effect of the Measurement Range}

Measurement range of optical systems (in lateral and vertical dimensions) is an essential factor to characterize the performance of the instrument. Additional to the applied acquisition algorithms, the accuracy of $X Y$-stage restricts the lateral information, whereas the performance of piezo or linear stages is one of the limiting factors in the quality of vertical information.

One common method to characterize vertical performance is the measurement of step height standards. But it should be taken into account that, this approach is useful only for the chosen step height. If the location of the measurement range in which the real measurement is carried out unknown, which is the case in most of the practical applications, the whole measurement range should be investigated.

In order to show this issue an experiment is carried on a $50 \mu \mathrm{m}$ step-height standard which is calibrated by PTB. This sample is measured by a white light interferometer which has a 400 $\mu \mathrm{m}$ measurement range by using a piezo. This $50 \mu \mathrm{m}$ height is measured on different locations within the measurement range. In other words, along the $400 \mu \mathrm{m}$ range different regions of " 50 $\mu \mathrm{m}$ " are chosen and 10 repeating measurements (temperature $20^{\circ} \mathrm{C} \pm 1^{\circ} \mathrm{C}$ ) are performed. Result of these experiments and the standard deviations are shown in figure 2.

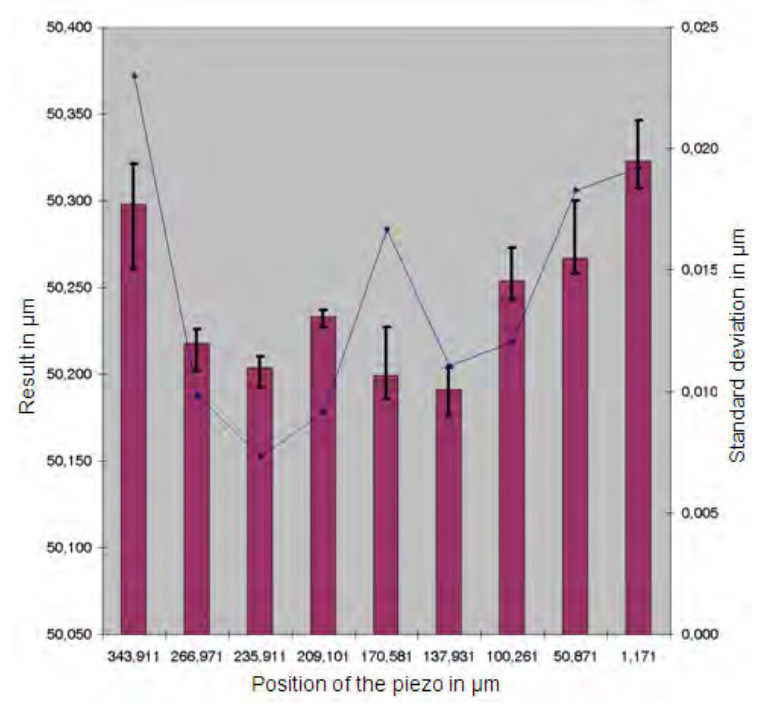

Fig. 2. Results of step height measurement along the vertical measurement range 
As it can be seen, the results of step height measurements depend on the location of the piezo position. This may be explained by the non-linearities in the measurement range of the instrument. Although the calibration process is specified by the manufacturer with a step height standard $(5 \mu \mathrm{m}$ or $50 \mu \mathrm{m})$, this vertical distance is insufficient to characterize the whole range. Unless the whole range is calibrated, there will always be some questionable points during the real measurement task.

The user should be aware of this point. A "50 $\mu \mathrm{m}$ " region along $400 \mu \mathrm{m}$ piezo may be calibrated; however the measurement can take place at any region within the measurement range.

\subsection{Effect of Topographic Properties of the Sample}

Another important aspect is the surface properties of the sample. Especially the roughness of the surface affects the signal quality which is returned from the measured region.

As stated in [3] the acquired topography is strongly affected if the surface is "rough" or "smooth". This has also been experimentally investigated in [4]. Different spheres with different roughness values are measured with an optical method. The results show that not only the slope of the sphere but also the roughness of the material strongly affects the signal quality. So it is not only enough to analyze the instrument with a single sphere, but its roughness value should also be considered.

\subsection{Effect of the Measurement System Resolution}

Surface metrology deals with both lateral and vertical dimensions, so it is important to characterize the resolution in both aspects. However resolving capabilities show huge differences in vertical and lateral directions. Because of this reason, it is not easy to specify them under a single term, like "resolution".

Height resolution is currently being discussed in ISO TC 213 WG16 in order to define the capability of an instrument to distinguish different features on surfaces. Due to the restrictions in the availability of the standards to test the vertical resolutions, manufactures' specifications mostly based on the experimental values, like multiplying the noise of the system with a constant. Although there are different approaches to specify it, the desired way is the development of a procedure in order to test it experimentally. As stated in [3] the vertical resolving power of metrology instruments is relatively small compared to other contributions to the uncertainties such as amplifications errors and noise. This fact makes it possible to conclude that the limiting factor in the structure resolution is not the vertical revolving capability of the instrument but its lateral resolution. However characterization of lateral information should be considered in more details.

\subsubsection{Case Study}

In order to understand the significance of lateral resolution, it is helpful to consider the following case study.
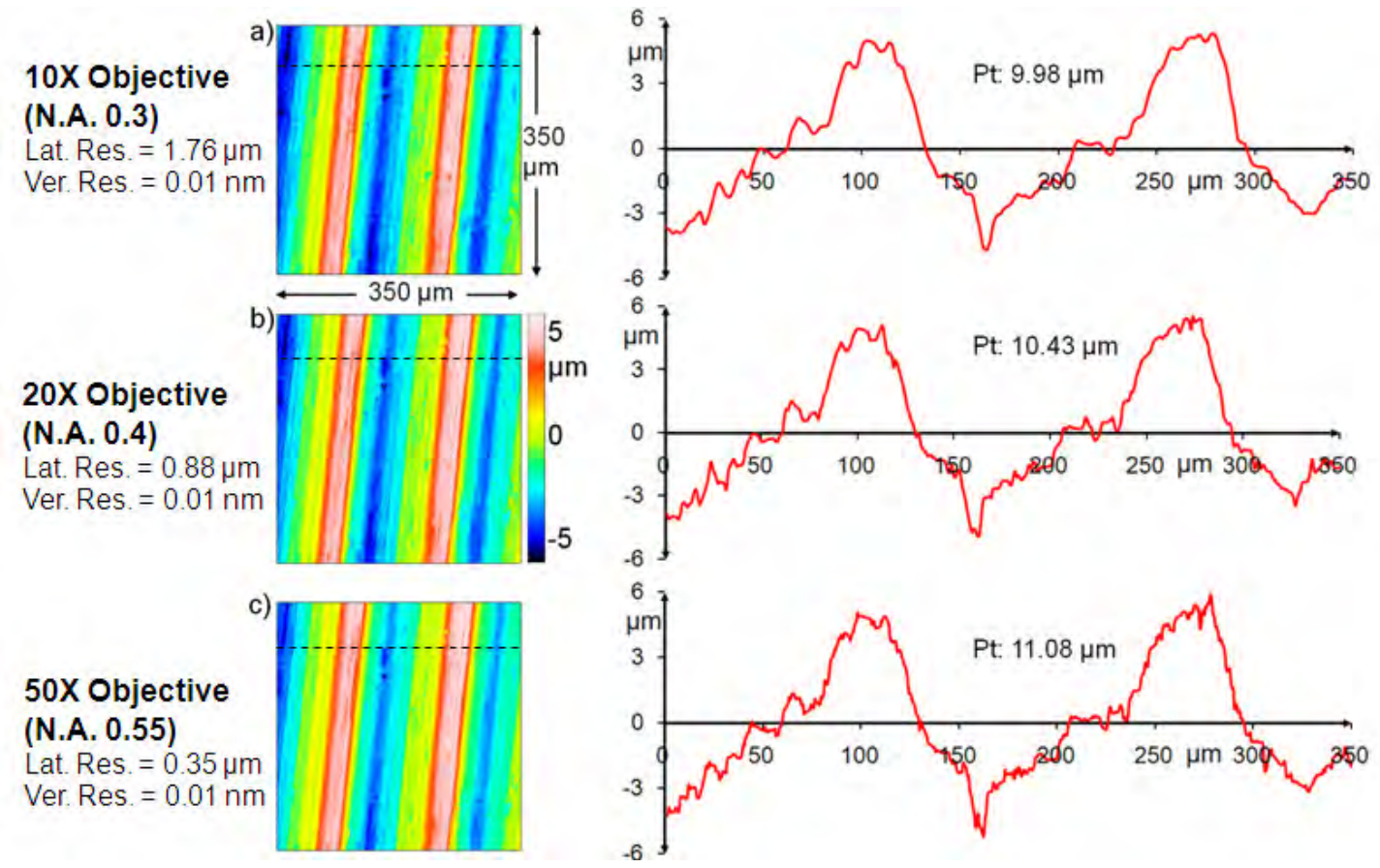

Fig. 3. Measurement of a ground surface with different objectives of a white-light-interferometer 
In this example a ground surface is acquired by a white light interferometer by using different objectives. The same part of the surface is measured with three different lateral resolutions. As seen in figure 3 , a $10 \mathrm{X}$ objective with a nominal lateral resolution of $1.76 \mu \mathrm{m}$, a 20X objective with a lateral resolution of 0.88 $\mu \mathrm{m}$ and a $50 \mathrm{X}$ objective with a resolution of $0.35 \mu \mathrm{m}$ are applied.

During the investigations, vertical resolution is kept constant at $0.01 \mathrm{~nm}$. As seen in figure 3, although there are some small differences in the structural details, the general run of the profiles look very similar. But the most important difference can be noticed if the calculated $\mathrm{Pt}$ values (total height of the profile) are also compared. Actually definition of $\mathrm{Pt}$ depends on the vertical information. Because of this reason at a constant vertical resolution it may be expected to have very close $\mathrm{Pt}$ values even the lateral resolution is changed. But as seen in figure 3 , calculated height information shows deviations, see the values of $\mathrm{Pt}$.

Based on this case study, it can be said that, degree of the vertical details depends not only on the vertical resolution but also on the lateral resolution. Because of this reason characterization of lateral resolution is very important in the evaluation of surface data.

However there is no agreed, specific definition of lateral resolution for areal instruments [3],[5]. And methods proposed in the mentioned guidelines are not in a state to characterize it in a task related way. Concepts like the "Siemensstar" can be used to get more information about the resolving power of the instrument.

\subsubsection{Concept of Siemens-Star}

As described in [6], [7] and [8] 3D Siemens-Star can be easily used to evaluate the lateral resolving power of instruments in surface metrology, which is not in that way possible with available standards and guidelines.

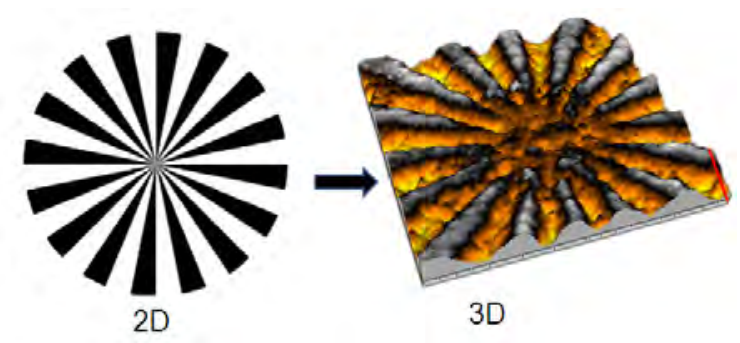

Fig. 4. Concept of 3D Siemens-Star

The idea of the well-known Siemens-Star has been modified and applied to the measurement devices in micro- and nanotechnologies. The developed structure has been named "3D Siemens-Star". In this new concept, as seen in figure 4, dark and white colors of the known Siemens-Star are substituted with grooves (peaks and valleys).

The basic idea of the evaluation method depends on the detection of the ambiguous region which is seen in the center of measured data (figure 5). This area describes the region, up to which the structure can be resolved. Changes in the number of peaks and valleys in the height profile and the changes in the measured height value have been set as the evaluation criteria. Detailed description is given in other investigations like [7] and [8].
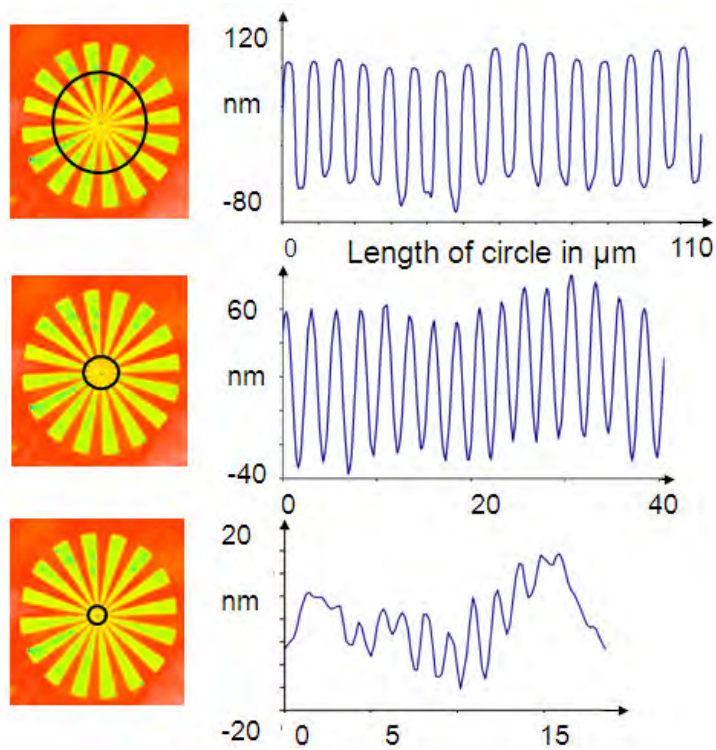

Fig. 5. Representation of the ambiguous region

This simple method provides the user possibilities to investigate the instrument performance in an experimental way.

By using such a structure, resolution power of used white-light-interferometer has been experimentally investigated by 10 repeating measurements.

Tab. 1: Results of the experiments with $3 D$ Siemens-Star

\begin{tabular}{|c|c|c|}
\hline $\begin{array}{l}\text { Objec } \\
\text { tives }\end{array}$ & $\begin{array}{l}\text { Lateral } \\
\text { Resolution in } \\
\mu \mathrm{m} \text { (by } \\
\text { manufacturer) }\end{array}$ & $\begin{array}{l}\text { Exp. lateral } \\
\text { resolution in } \mu \mathrm{m} \\
\text { (with 3D Siemens } \\
\text { Star) }\end{array}$ \\
\hline $10 \mathrm{X}$ & 1.76 & $1.92 \pm 0.07$ \\
\hline $20 \mathrm{X}$ & 0.88 & $1.02 \pm 0.04$ \\
\hline $50 \mathrm{X}$ & 0.35 & $0.52 \pm 0.01$ \\
\hline
\end{tabular}


As seen in table 1, all the calculated results are higher than the specified values and this shows that the measurement method differs from the theoretical values. This is also the reason to find out a practice-oriented method in order to get information about the resolution performance of different instruments.

\section{Conclusion}

Measuring the metrological properties of the instruments is essential to understand and characterize the behavior of the instrument and to have traceable results. Although they are not completed, there are many scientific activities to standardize such methods. However they will not characterize the instrument completely for the real measurement task in which technical surface (rough surfaces) is analyzed. On the other hand there are some capability analysis methods like MSA, but in such guidelines instrument specific properties are not completely covered. But from the user point of view, examples are required to understand the limitations of the instruments. Furthermore it is crucial to know about the influencing factors of the calibration. Based on those needs, this paper presents some of those factors which are not completely considered during calibration of the instrument.

Presented experimental investigations show that topography of the measured surface, measurement range and also the resolving power of the instrument are essential when measuring rough surfaces. Idealized surfaces of standards are not enough to test the performance of the instrument. The presented results show that additional effort is required to characterize such properties. It is needed to develop additional methods, like 3D SiemensStar, in order to characterize the metrological properties of the system. Such attempts are needed to make the end user to be aware of the limits of the instrument.

\section{Acknowledgments:}

The author would like to thank to Prof. Dr.-Ing. A. Weckenmann, who made most of the research possible in the chair QFM.

\section{References}

[1] VDI/VDE 2655: Optical measurement and microtopographies - Calibration of interference microscopes and depth measurement standards for roughness measurement

[2] QS 9000: Measurement System Analysis, Reference Manual, Third edition
[3] R. Leach, Optical measurement of surface topography, Springer 2011. -ISBN 978-3642-12011-4

[4] W. Ehrig, U. Neuschaefer-Rube, T. Birth, Prüfkörper zur Untersuchung des Werkstückeinflusses auf hochgenaue Koordinatenmessungen mit optischen Abstandssensoren; Neue Strategien der Mess- und Prüftechnik für die die Produktion von Mikrosystemen und Nanostrukturen, Abschlussbericht DFG Schwerpunktprogramm 1159 StraMNano,154-163 (2011), ISBN 978-38440-0358-1

[5] M. Senoner, T. WIRTH, W.E.S. UNGER, Imaging surface analysis: Lateral resolution and its relation to contrast and noise, Journal of Analytical Atomic Spectrometry 25 (2010) 9, p. 1440-1452.

[6] Ö. Tan, Characterization of Micro- and Nanometer Resolved Technical Surfaces with Function-oriented Parameters, Erlangen-Nürnberg, Univ. thesis, 2012

[7] A. Weckenmann, Ö. Tan, J. Hoffmann, Z. SUN, Practice-oriented evaluation of lateral resolution for micro- and nanometer measurement techniques, Measurement Science and Technology 20 (2009) 6, 065103 (8pp).

[8] A. Weckenmann, Ö. Tan, L. Shaw, N. Zschiegner, Comparison of resolution specifications in micro- and nanometer measurement techniques, AMA-Service $\mathrm{GmbH}$ (Publ.), Proceedings of the Sensor+Test Conference 2009 "Sensor 2009" (Nuremberg, Germany, 26.28.05.2009), Volume 2, p. 377-382. 